\title{
Single Frequency WAAS Augmentation Observations (L1 vs. L5) on a Ground Based GPS L1 C/A Solution
}

\author{
Marc-Antoine Fortin, Jean-Christophe Guay, René Jr. Landry \\ École de Technologie Supérieure (ÉTS), Montréal, Canada \\ Email: Marc-Antoine.Fortin@lacime.etsmtl.ca, Jean-Christophe.Guay@lacime.etsmtl.ca, \\ rene.landry@etsmtl.ca
}

Received 12 June 2014; revised 10 July 2014; accepted 8 August 2014

Copyright (C) 2014 by authors and Scientific Research Publishing Inc.

This work is licensed under the Creative Commons Attribution International License (CC BY). http://creativecommons.org/licenses/by/4.0/

(c) (i) Open Access

\section{Abstract}

This paper presents observations on the WAAS L1 and L5 signals quality and their impact on the robustness of the navigation solution by quantifying the contributions of each broadcasted differential correction. This work is undertaken with the intent of defining performance benefits of L5 by dual frequency WAAS users and is to provide useful material for Minimum Operational Performance Standard (MOPS) development. In this perspective, a study of the WAAS signal characteristics is first carried out. The information gathered is then used to compare various GPS solutions in terms of frequency diversity, satellite diversity, pseudorange noise and different signal corrections and their impacts. These solutions are compared against a reference standalone GPS solution. All statistics are computed with respect to a post-processed Novatel Waypoint Real-Time Kinematics (RTK) GPS L1/L2 semi-codeless static solution, considered as the reference. A discussion on some simplifications with respect to specifications (i.e. MOPS) that could be considered by receiver manufacturers closes the paper. It is confirmed that the current WAAS navigation message definition is the same on both the L1 and L5 frequencies, the latter further being Manchester coded, thus avoiding data ambiguity. The $+5 \mathrm{~dB}$ SNR on L5 has minor impacts in terms of reliability and continuous availability in the presented scenarios, but would become especially beneficial in hostile environments, despite a greater number of pulsed interferers. Another demonstration is that the WAAS message varies slightly from one WAAS satellite to another, even if corrections are generated centrally. Finally, it is observed that WAAS and GPS signals pseudorange noise are comparable on a "per frequency" basis.

\section{Keywords}

GPS L1, WAAS L1, WAAS L5, MOPS, Pseudorange Noise 


\section{Introduction}

The civil international aircraft industry constantly seeks for satellite navigation performance improvements. Following that thread, the first Space Based Augmentation System (SBAS), namely the Wide Area Augmentation System (WAAS), was declared operational on L1 (1575.42 MHz) in 2003, already a decade ago. It is now known that WAAS signals improve GPS signal processing by correcting ionospheric delay errors, ephemeris differential corrections (including clock bias/drift and GPS satellite position), resulting in a greater solution integrity and accuracy. Initially, SBAS was intended to improve accuracy and provide integrity assurance. In its earliest form, it did not even include differential corrections [1]. In time, the most obvious effect of SBAS became to annihilate the Selective Availability (SA), which could be differentially corrected [2]. This intended degradation of the signal quality with military purposes has been discontinued since May 2000; new GPS III satellites should no longer include SA capability, since it has been permanently deactivated [3]. Fortunately, since SA has included a voluntary clock dither error, SBAS systems still compensate for involuntary GPS clock errors due to hardware anomalies onboard Space Vehicles (SV). A complete error source assessment may be found in [4]. Because of its application in flight industry, security issues impose long validation cycles. For other applications, the latest 8-year-old Minimum Operational Performance Standard (MOPS) [5] could be re-visited in terms of general SBAS implementation rules with an up-to-date environment (e.g. considering SA has completely been deactivated and will not come back). This is important since GPS L1 cannot satisfy performance required by Federal Aviation Administration (FAA) for various phases of flight on its own, especially for approach with vertical guidance.

This assessment is even more important since WAAS has been continuously undergoing improvements and operational capability upgrades as part of its four phases [6]:

1) Initial Operating Capability (IOC) accomplished in 2003;

2) Full LPV Performance (FLP) completed in 2008;

3) Full LPV-200 Performance extended through 2013;

4) Dual Frequency Operations (DFO) planned until 2044.

Phase 1 provided high availability "in route" through non-precision approach such as Lateral and Vertical Navigation (LNAV/VNAV) service over the Conterminous United States (CONUS) as well as limited Localizer Performance with Vertical Guidance (LPV) approach service. Phase 2 provided full LPV service with a limited LPV-200 approach service availability within CONUS. Phase 3 provided robust, reliable, and sustainable LPV-200 capability and coverage throughout CONUS. This was very welcome during solar maximum, i.e. the 11-year cycle peak that last occurred in 2013 [7] [8]. In November 2011, the FAA approved its first Operational Specifications for Required Navigation Performance (RNP) Authorization Required (AR) using WAAS. Moreover, NAV CANADA has 180 approaches pending design and publication at 92 airports, on top of the already compliant 36 airports with a total of 57 approaches published [9]. U.S. WAAS now counts 38 ground reference stations, achieving $99 \%$ availability over the main land. 1900 U.S. airports have commissioned 3000 LPV precision approaches and 5000 are expected by 2016. Phase 4 should implement the paradigm change where GPS L5 would replace L2 $\mathrm{P}(\mathrm{Y})$ in WAAS ionosphere computation; i.e. WAAS would then use L1 and L5 to generate the necessary estimates for Single Frequency Users (SFU) on L1, while Dual-Frequency Users (DFU) would calculate ionosphere induced delays directly through L1 and L5. While the current 3-GEO (Inmarsat-4GEO-PRN 133, Anik-F1R-PRN 138 and Galaxy 15-PRN 135) constellation is already dual-frequency capable, the WAAS L5 Signal In Space (SIS) is only used for line of sight ionosphere measurements. In addition, new GEO satellites are planned for procurement around 2015-2018 as current WAAS constellation leases will expire in this timeframe.

All this activity, as well as the introduction of the L5 (1176.45 MHz) civil signal on all GPS Block IIF (and newer) satellites, planned to launch on a replacement-basis, opens new possibilities for use of the WAAS system. WAAS transmissions improve system accuracy: 1 ) by reducing ranging measurement errors through the transmitted differential corrections for each GPS satellite and 2) by improving geometry by making their ranging signals available in addition to the set of GPS measurements. The latter is possible since the WAAS signal is synchronized with GPS system time [10]. Frequency diversity would also be very helpful in case of RF Interference (RFI) in one of the used frequency bands [11], although phase 4 includes no plans to provide for L5-only users. So once the current WAAS transmissions will be updated, there would be no more frequency diversity available in the event of RFI. 
The two Aeronautical Radio Navigation Services (ARNS) L band frequencies used in WAAS, among the four Radio Navigation Satellite Services (RNSS), not only grant frequency diversity to aircrafts, but also offer GPS receiver manufacturers a lot of design choices for their future products. Hence, the purpose of this paper is to provide an overview of the actual status of WAAS signal precision and integrity on both L1 and L5, although there are no plans for a WAAS L5 service; current WAASL5 signals are not officially declared online by the FAA [12]. The WAAS signal characteristics are summarized hereafter before the measurements integration into the solution is described, and its tests methodology is defined. The following is a study of the WAAS signal quality and contents. Then, a robustness analysis of different WAAS signals is presented before a short discussion and conclusive remarks.

\section{WAAS L1/L5 Signals Characteristics}

There are two satellite signals currently broadcasted by the WAAS system: one on L1 and another on L5. They are both modulated with Binary Phase Shift Keying (BPSK) on their respective center frequencies, shared with GPS. As opposed to the GPS L5 civil signal, WAAS L5 has no quadrature component [13]. Table 1 summarizes WAAS signals' general characteristics [14] [15].

The structure of the WAAS message data block is composed of an 8-bit partial preamble (of a 24-bit long preamble spread onto three consecutive data blocks), a 6-bit message type identification and a 212-bit data field, followed by a 24-bit Cyclic Redundancy Check (CRC) parity word. Each 250-bit data block is encoded through rate-1/2 Forward Error Correction (FEC) based on a 7-bitconstraint length, resulting in 500 symbols. It is noticeable that there is no composition difference between WAAS L1 and WAAS L5 messages at this point, apart from the WAAS L5500 sps being Manchester coded, resulting in twice the processing bandwidth at $1 \mathrm{kSps}$. This Manchester coding could also be looked at a 2-bit secondary code (i.e. 1.0) [16]. The occupied signal bandwidth however remains at least $20 \mathrm{MHz}$, such that at $95 \%$ of the broadcast power is contained within $\pm 12 \mathrm{MHz}$ around at the broadcast frequency. Experimentation further shows that the WAAS L1 to L5 symbols difference is below $0.1 \%$, confirming that the same navigation message is currently being broadcasted from a given satellite simultaneously on both its WAAS signals, as mentioned in [16].

Furthermore, 63 message types are possible in WAAS L1, but only 18 are defined in its specifications ([17], p. 12). This limited message set is currently also used as a placeholder for WAAS L5 while it remains to be defined as part of the dual frequency L1/L5 MOPS development.

\section{Test Methodology for WAAS L1/L5 Analyses}

Initially, a post-processed zero-baseline Real-Time Kinematic (RTK) method was applied on two Novatel DL4Plus receivers with L1/L2 semi-codeless solutions in order to establish the true reference coordinates of the antenna used for the tests presented herein. A passive Novatel-704 antenna, placed on the ÉTS roof with clear visibility, has been used with a proper inline amplification chain for all the tests. The Waypoint software provided a solution with fixed ambiguity for all samples, i.e. the best achievable solution quality. These reference coordinates (i.e. $45.494035^{\circ}$ latitude, $-73.562770^{\circ}$ longitude and $10.445 \mathrm{~m}$ elliptical height obtained with sub-millimeter standard deviation) are later used to compute all the statistics presented in this paper.

A research prototype of a universal GNSS receiver (RxGNSS) was used to conduct the tests presented in the following sections. Currently, 12 channels can be seamlessly configured to acquire and track any civil GNSS or

Table 1. General characteristics of WAAS signals.

\begin{tabular}{llccc}
\hline \multicolumn{2}{c}{ WAAS signals } & L5 & L1 \\
\hline Carrier frequency & $(\mathrm{MHz})$ & 1176.45 & 1575.42 \\
Code length & (chips) & 10230 & 1023 \\
Code rate & (Mcps) & 10.230 & 1.023 \\
Code duration & $(\mathrm{ms})$ & 1 & 250 \\
Data rate & (bps) & (sps) & 500 & 250 \\
Encoded symbol rate & 20 & 500 \\
3-dB Bandwidth & $(\mathrm{MHz})$ & & 20 \\
\hline
\end{tabular}


SBAS signal [18]. The RxGNSS further includes three configurable RF front-ends connected to a Lyrtech VHSADAC board with 8 ADCs and 1 XC4VSX55-10FF1148 FPGA (with an embedded MicroBlaze processor) as well as an external computer [19]-[22]. In order to appreciate the impacts of WAAS corrections, a GPS L1 C/A code solution with carrier smoothing is considered as the performance benchmark.

All three WAAS satellites are visible from the antenna location, as described in Table 2. As a final note, the reader should be aware that slow, fast and ionospheric corrections were applied to the GPS L1 C/A signals being tracked, for all WAAS solutions presented below. All logs are described as the tests below are defined.

\section{Analysis of the WAAS L1 and L5 Signals Characteristics}

Tests were performed with the RxGNSS to compare WAAS signals on L1 and L5 in terms of C/ $\mathrm{N}_{0}$, navigation message contents, pseudorange noise and resulting navigation solution performances.

\subsection{C/NO Impacts Analysis}

MOPS states that it is often sufficient to choose the WAAS satellite with the greatest elevation angle, but warns that it may not be the best [23]. At the time the tests were conducted, PRN 133 (with highest elevation angle) was declared Non Precision Approach (NPA); PRN 138 was therefore used by default as it provided higher signal strength. The transmitted power level is mainly determined by the satellite Equivalent Isotropically Radiated Power (EIRP), although there could be spatial variation between the L1 and L5 arrays. A detail worth noting is the WAAS L5 signal strength for PRN 138 was witnessed to be more than 4dB higher than on L1, which differs from previous findings where both frequencies showed similar $\mathrm{C} / \mathrm{N}_{0}$ (within $\pm 1 \mathrm{~dB}-\mathrm{Hz}$ ) [24]. This also goes against the Signal In Space (SIS) power levels contained in the WAAS Technical Specifications for GEO 5/6/7 Service Lease where the maximum transmitted power is $2.5 \mathrm{~dB}$ higher on L5 compared to L1 [15]. One could have expected higher L5 signal power to help fight against the known pulsed interference environment L5 resides in. Indeed, Distance Measuring Equipment (DME), Tactical Air Navigation (TACAN), Joint Tactical Information Distribution System (JTIDS) and Multifunctional Information Distribution System (MIDS) all represent a higher worldwide pulsed threat than in the other 3 RNSS L bands [25].

From the same test performed on June 20, 2012 used in the previous paragraphs, a $\mathrm{C} / \mathrm{N}_{0}$ statistical analysis was also conducted, the results of which are summarized in Table 3.

The $~ 5.44 \mathrm{~dB}-\mathrm{Hz}$ increase of L5 over L1 for PRN 138 has multiple advantages, such as in tracking loops performance (i.e. reduced code and phase thermal noise provides better solution accuracy, assuming their pseudoranges were included in the solution), as well as a greater navigation message successful decoding ratio (i.e. fewer tracking losses).

Furthermore, MOPS receivers are required to have no worse than a $0.05 \%$ message loss rate at the minimum power level; i.e. the end-to-end SIS service reliability must be greater than $99.95 \%$ in any one hour interval [5]. In the RxGNSS, the CRC pass rate translates into a successfully received messages ratio. In the present case, a

Table 2. WAAS satellite relative location (from Montréal, Canada).

\begin{tabular}{|c|c|c|c|c|}
\hline \multirow{2}{*}{ PRN } & \multicolumn{2}{|c|}{$\mathrm{C} / \mathrm{N}_{0}(\mathrm{~dB}-\mathrm{Hz})$} & \multirow{2}{*}{ Elevation ( ${ }^{\circ}$ ) } & \multirow{2}{*}{$\begin{array}{c}\text { Azimuth } \\
\left(^{\circ}\right)\end{array}$} \\
\hline & $\mathrm{L} 1$ & L5 & & \\
\hline $133^{\mathrm{a}}$ & 40.0 & 42.0 & 35.5 & 213.7 \\
\hline 135 & 37.4 & 39.4 & 12.5 & 247.3 \\
\hline 138 & 40.9 & 46.1 & 28.2 & 223.3 \\
\hline
\end{tabular}

a. PRN 133 is Non-Precision Approach (NPA) on June 19 (th $^{\text {th }} 2012$.

Table 3. C/N0 statistics for satellite PRN 138.

\begin{tabular}{cccccc}
\hline Signal & Mean & Std. & Min. & Max. \\
\hline L1 & 40.8 & 0.2 & 40.1 & 41.5 \\
L5 & 46.2 & 0.4 & 44.9 & 47.0 \\
\hline
\end{tabular}


CRC pass rate of $99.6 \%$ was achieved on L1, and $100 \%$ on L5. Isolated discontinuities on L1 were caused by tracking losses due to lower signal strength. In order to maximize reliability, the following tests are only conducted with WAAS L5 corrections, tracked with high signal strength.

The WAAS navigation message is composed of two symbols per data bit, resulting in 500 sps. The RxGNSS samples at each $1 \mathrm{~ms}$ long spreading code period, providing exactly two samples per symbol. Hence, a samples pair (even and odd) is obtained for every encoded WAAS symbol transmitted.

In the case of WAAS L1, a $1 \mathrm{kSps}$ stream error is defined as different even and odd samples within a symbol. As expected, the error count increases as the signal strength falls under $40 \mathrm{~dB}-\mathrm{Hz}$, most probably due to tracking losses. Nevertheless, such a stream error rate $(\bar{x} \pm 3 \sigma)$ of $1.1 \% \pm 2.0 \%$ at $38 \mathrm{~dB}-\mathrm{Hz}$ would still remain acceptable, considering that Viterbi decoding resists to 15 distributed errors per 500 -symbol data blocks (i.e. $3 \%$ of all symbols), $78.55 \%$ of the time, as simulated in [26]. Note that these numbers do not account for FEC gains, which would result in greater error rate tolerance at a given bit energy per noise density $\left(E_{b} / \mathrm{N}_{0}\right)$. Indeed, the Word Error Rate (WER) is specified to be $1 \times 10^{-3}$ [5]. On the other hand, GPS L1 C/A stream errors can easily be averaged out over 20 consecutive samples. Such stream errors (here defined as different samples within a 20 sample long bit) were not witnessed on the RxGNSS at signal strengths above $41.5 \mathrm{~dB}-\mathrm{Hz}$, which is consistent with observations on the WAAS signals. In the case of GPS L5, the secondary code may still be mapped to one of its two polarity, whichever is closest to the one observed, once synchronization is achieved.

Considering that two $1 \mathrm{kSps}$ samples represent one 500 sps WAAS symbol (for both WAAS L1 and L5), a common WAAS decoding approach is to consider only one sample out of two. For such an approach to work, both alignments (odd and even) need to be tested in order to achieve initial alignment. This simple approach allows maintaining complete frequency diversity with the current WAAS signal definition. It has thus been applied to the following tests.

\subsection{Analysis of the WAAS Navigation Message}

Figure 1 displays a navigation stream auto-correlation. This 3-second periodicity is partly due to the WAAS 24-bit preamble being distributed over 8-bit preambles on three consecutive 1-s long data blocks. However, 8 bits out of 250 may not be sufficient to justify normalized correlation levels larger than $50 \%$. The occurrence of each message type occurrence is bounded by its maximum update interval, defined as a multiple of $60 \mathrm{~s}$ in most cases. The User Differential Range Error Index (UDREI) data, bared by message types 2 - 6 and 24, is the only one with a $6 \mathrm{~s}$ maximum update interval; a $3 \mathrm{~s}$ repeat period would thus seem to be a safe operation mode, providing a more probable reason for the witnessed 3 s periodicity of the navigation message.

WAAS satellites may broadcast messages from any WAAS Master Station (WMS). In general, each GEO broadcasts information from a different WMS. However, there are times when one WMS may be broadcasting

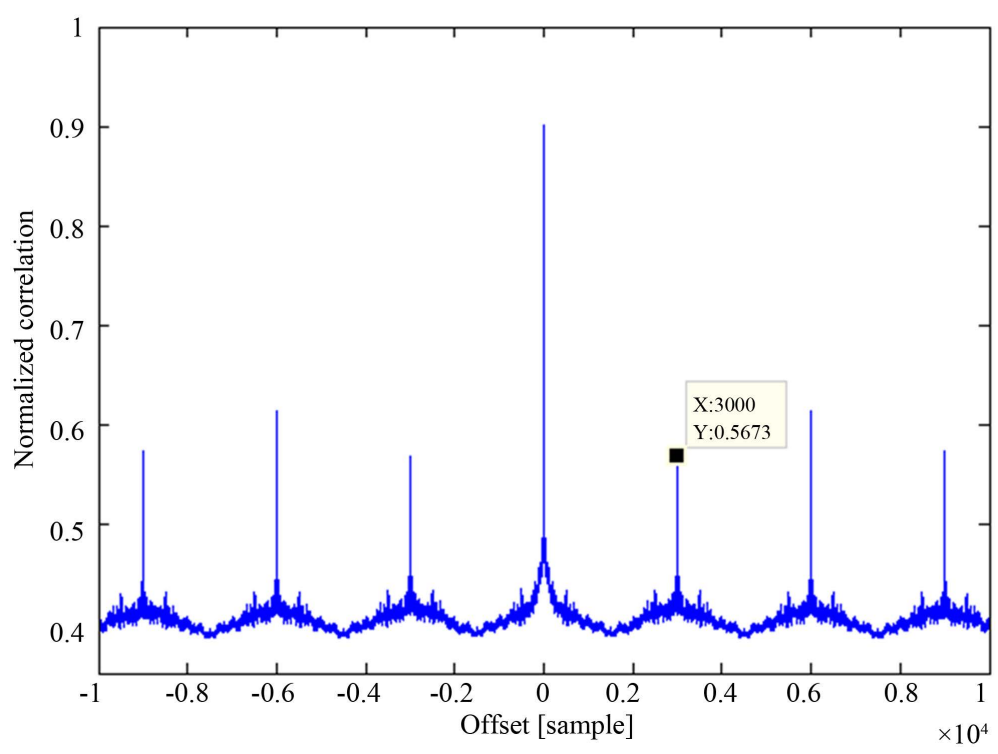

Figure 1. Zoom on a partial auto-correlation showing a 3 s periodicity. 
through more than one GEO. Also, all three master stations have the same input data and therefore the message content among the WMSs is very similar, but not necessarily identical. We formulated the hypothesis that different WAAS satellites transmitting different contents could influence the resulting navigation solution. In order to evaluate the impact of WAAS corrections provided by different satellites, two other tests were realized, both performed on June 20, 2012. For each test, all the three WAAS satellites were assigned to a tracking channel and their sampled $1 \mathrm{kSps}$ stream were compared. More precisely, the encoded navigation message symbols are $>45 \%$ different from one satellite to another. Although Forward Error Correction (FEC) provides a data decoding robustness, it also propagates a small data difference over several symbols [27].

This falsely increases the contents difference ratio when comparing symbol streams from different satellites. Bearing in mind that message types 9, 12 and 28, respectively for the broadcasting satellite ephemerides, clock and their covariance, will vary from one satellite to another (over the complete data field and thus on the CRC too) [28], a smaller symbols difference ratio was expected. Indeed, these message types maximum update intervals are respectively 120, 300 and $120 \mathrm{~s}$, which is rather slow compared to those of other message types. These other message types should be similar in contents, although they could be transmitted in a different order or at a different time.

Also, because of the navigation message structure, missing a data block could have noticeable consequences. For example, if the Ionospheric Grid Point (IGP) Mask Message Type 18 (defining the way to interpret the following Ionospheric Delay Corrections Messages Type 26) first reception was missed, ionospheric corrections would not be available until a new Ionospheric Mask Issue of Data (IODI) is received. The IODI changes very infrequently (less than once a year) so a non-aviation user could apply an even longer time out and ensure that the ionospheric corrections are properly decoded based on previous data. In cold-start mode, an extra augmentation setup delay could last up to a maximum update interval of $300 \mathrm{~s}$ for Message Type 18. In the long run, this threat is minimized through the Message Type 18 time out interval of 1200 s. Indeed, after first successful reception, such an outage would imply Message Type 18 is missed four times in a row. Note that aviation procedures are interrupted whenever outages last more than $4 \mathrm{~s}$.

Similarly, in cold start mode, missing the first PRN Mask Message Type 1 could cause an outage lasting up to $120 \mathrm{~s}$ for all of the following message types (assuming a missed change in IOD PRN (IODP) mask) [28]:

- Fast Corrections (Message Types 2 - 5 and 24);

- Integrity Information (Message Type 6);

- Acceleration Information (Message Type 7);

- Long-term Corrections (Message Type 25);

- Clock-ephemeris Covariance (Message Type 28).

Again, in the long run, one would need to miss five consecutive Message Type 1 data blocks to suffer such an outage.

\subsection{Pseudorange Noise Analysis}

Another useful metric to consider when seeking for the best WAAS signal to use is the pseudorange noise. The pseudorange being proportional to the propagation time (4.1), it should behave approximately as a parabola, from horizon to zenith and horizon again. Its second derivative should thus tend towards a constant value. The pseudorange noise - a random process with an order greater than 2-could then be approximated as the remaining variations of the pseudorange second derivative (4.2) [29]. At that level, only the chip index and the phase of the chipping rate clock signal, taken at $1 \mathrm{kHz}$, need to be considered for a static receiver ([26], p. 264). This simplification is useful in analyzing WAAS signals, as the associated navigation message does not provide a readily available transmission time such as the GPS Time of Week (TOW); SBAS plans for time information in message type 12, which was not transmitted by WAAS satellites in 2012. Fixed ambiguity may be solved for WAAS pseudorange based on a pre-existing navigation solution, and remains valid as long as the WAAS signal is continuously pursued. Combined with GPS L1 C/A signals, it is however possible to extrapolate that information since both data blocks are synchronized, although WAAS time is offset with respect to GPS time. Moreover, L1 and L5 being characterized by different paths, an extra time offset is to be considered [23]. Noise is then quantified as the standard deviation of the pseudorange second derivative $\sigma_{n_{P R}}$ :

$$
T_{\text {prop. }}=N_{\text {code }} \cdot T_{\text {code }}+\frac{N_{\text {chip }}+\Theta_{\text {chip }}}{f_{\text {chip }}}[\mathrm{s}]
$$




$$
n_{P R} \propto \ddot{T}_{\text {prop. }}=\frac{\frac{\partial^{2}}{\partial t^{2}}\left\{N_{\text {chip }}+\theta_{\text {chip }}\right\}}{f_{\text {chip }}}\left[\mathrm{m} / \mathrm{s}^{2}\right]
$$

where:

$T_{\text {prop. }}$ is the propagation time;

$N_{\text {code }}$ is the number of complete code;

$T_{\text {code }} \quad$ is a complete code period;

$N_{\text {chip }}$ is the chip index, within a code;

$\theta_{\text {chip }} \quad$ is the phase of the chipping rate clock;

$f_{\text {chip }}$ is the chipping rate;

$n_{P R} \quad$ is the pseudorange noise;

A snapshot analysis shows the second derivative of the propagation time (C.f. Figure 2 where the legend indicates the signal type, its average $\mathrm{C} / \mathrm{N}_{0}$ and its noise standard deviation).

The lower propagation time noise is due to the WAAS L5 chipping rate being 10 times faster, thus allowing for 10 times narrower correlator spacing. Indeed, it is well known that code phase jitter performance depends on the slope of the discriminator curve and the SNR. More precisely, the Delay Lock Loop (DLL) has a closed loop 1-sigma error defined as [30] [31]:

$$
\sigma_{\varepsilon} \approx \frac{c}{f_{c}} \sqrt{\frac{2 \cdot B_{L} \cdot T_{P} \cdot R_{n}(0)}{K^{2}}}[\mathrm{~m}]
$$

where:

$C$ is the speed of light (i.e. 299, 792, $458 \mathrm{~m} / \mathrm{s}$ );

$B_{L} \quad$ is the loop bandwidth;

$T_{P} \quad$ is the integration time (i.e. $1 \mathrm{~ms}$ );

$R_{n}(0)$ is the noise correlation at null offset between the incoming and the replicate codes;

$K \quad$ is the discriminator slope $\equiv\langle S\rangle / \tau$;

$S$ is the useful signal;

$\tau$ is the offset between input signal and replica.

The impact of correlator spacing on different signals are compared in Figure 3. Note that the chipping rates are 1.023 Mcps for GPS L1 C/A and 0.511 Mcps for GLONASS. The correlator spacing is set to \pm 0.5 chip during acquisition (not shown) and 0.05chip during tracking for all L1 signals, but to \pm 0.17 chip for WAAS L5, due to

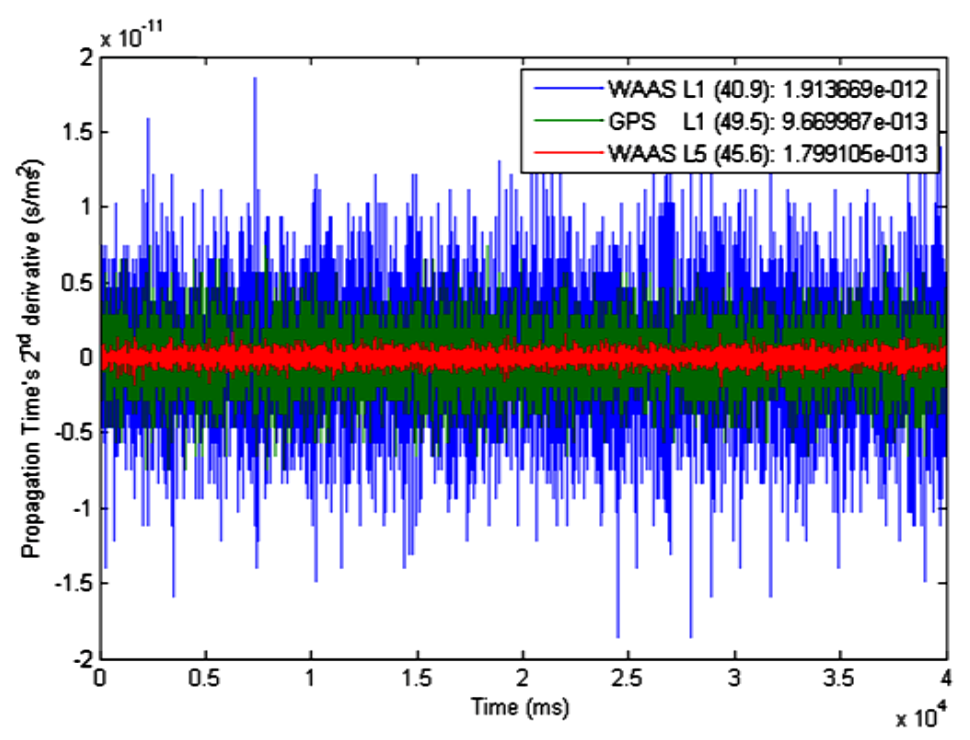

Figure 2. Propagation time noise during 40s (Legend $\Rightarrow<$ signal type $>$ $\left(<\mathrm{C} / \mathrm{N}_{0}>\right)$ : $<$ noise standard deviation $\left.>\right)$. 


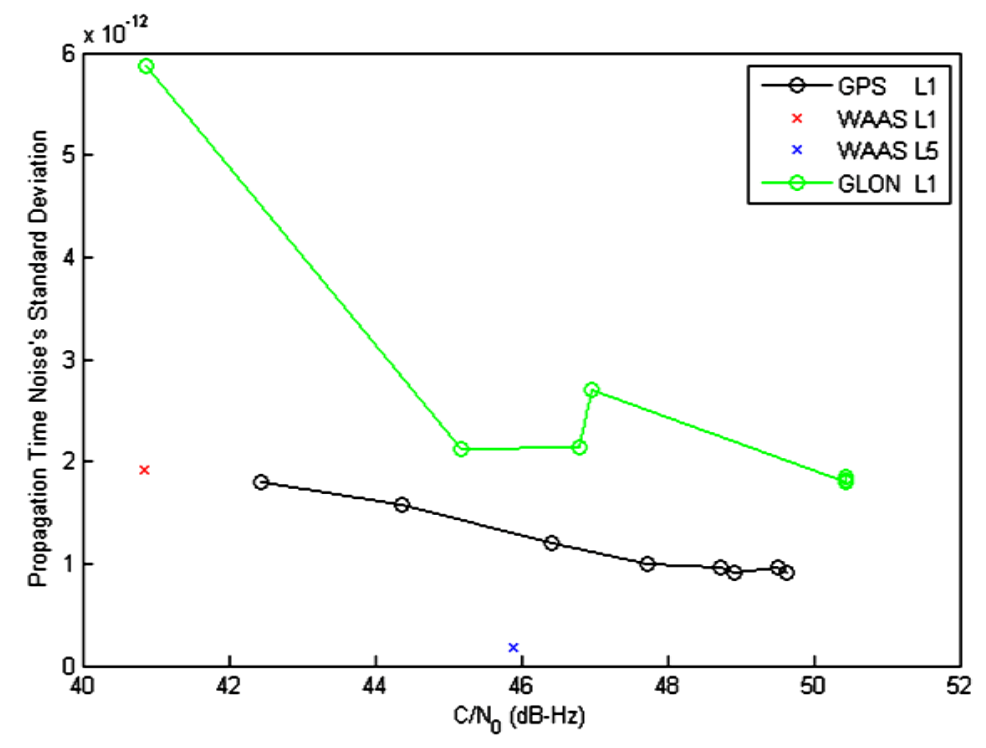

Figure 3. Correlator spacing impact on DLL noise vs. C/ $\mathrm{N}_{0}$.

a $60 \mathrm{MHz}$ sampling frequency limitation. From the figure, it can be seen that WAAS L1 quality is in line with that of GPS L1 C/A, while WAAS L5 appears 10 times better for a given $\mathrm{C} / \mathrm{N}_{0}$. Considering that the signals intrinsic phase noise can be dominant contributor to carrier and pseudorange measurement performance and that carrier tracking error may also contribute to measurement errors, it appears that WAAS signal pseudorange noise are similar with that of GPS signals on L1 and L5 [32] [33]. Indeed, WAAS has specified an end-to-end SIS single-sided phase noise vs. frequency offset from the nominal carrier [15], which provides better performance relative to the MOPS specifications of 0.1 radian error in $10 \mathrm{~Hz}$ tracking loop.

\subsection{Navigation Solution Performance Analysis for Different WAAS Satellites}

Two logs were taken in order to compare the WAAS satellite corrections impact on the receiver performances for a static user; no test was performed on satellite PRN 133 as it was NPA at the time the tests were conducted [34]. Test \#1 was performed on June 22, 2012 and lasted 24 hours, where a WAAS L5 channel was configured to track satellite PRN 138. Meteorological observations [35] for that day at the Montréal Dorval Airport, Canada (YUL) - $14.9 \mathrm{~km}$ away from the antenna as the crow flies-were a broken sky condition with an average temperature of $23.5^{\circ} \mathrm{C}$. Test \#2 was performed on June 23, 2012 and lasted 24 hours, where a WAAS L5 channel was configured to track satellite PRN 135. The sky conditions were scattered with an average temperature of $21.5^{\circ}$ C. Both tests started at 8 A.M. It can be noticed that PRN 138 has a greater elevation angle than PRN 135 relative to the antenna position, and thus its $\mathrm{C} / \mathrm{N}_{0}$ is consequently stronger (C.f. Table 2).

Table 4 highlights some of the statistical results. The performance differences are considered negligible: only a few centimeters difference which could be explained by a change in weather between the two tests. Hence, it can be concluded that, despite not exactly the same, WAAS message lead to equivalent, comparable differential positioning performances. Because of the stronger $\mathrm{C} / \mathrm{N}_{0}$, satellite PRN 138 has been chosen for the following tests aimed at quantifying the positioning impact of differential corrections on different WAAS signals.

\section{Differential Correction Comparison through WAAS L1 and L5}

Now that the WAAS signals have been characterized, different static tests are realized to analyze the robustness of a GPS solution with different WAAS options. All the 15 message types defined for WAAS L1 are decoded (from either WAAS L1 or L5 identical transmissions) and most of their data is applied after Carrier Smoothing (CS) of the raw GPS L1 C/A measurements, if they are to be significant, i.e. not buried in code measurement noise [26]. Basically, slow and fast corrections are respectively applied to GPS satellites clock and ephemerides, as well as to the raw pseudorange measured by the receiver, while ionospheric corrections replace the GPS broadcasted Klobuchar ionospheric model. These corrections should be extrapolated in time with degradation 
parameters. However, now that SA has been deactivated, Rapid Rate Corrections (RRC) can be removed in ground receivers (where integrity is not as critical as for air-born receivers) as it introduces $12 \mathrm{~s}$ periodic noise [2], and is thus not applied. In the navigation solution presented herein, WAAS almanacs and ephemerides are only used to populate the sky plot; their pseudoranges and the SBAS UTC data blocks are thus not used. Also, integrity is achieved by bounding the pseudorange measurements; out-of-bound measurements being discarded while others are weighted in the Kalman solution. Prior to analyzing the metrics below, positioning performance improvements in these conditions were assessed to be comparable to those obtained with a Novatel DL4-Plus receiver hooked up to the same RF setup.

In the following paragraphs, horizontal statistics are compared, i.e. standard deviations, mean errors, CEP and R95. More precisely, three sets of tests were conducted: WAAS corrections from L1 vs. L5; day vs. night and slow vs. fast corrections.

\subsection{WAAS L1 vs. L5 Corrections Impacts on Positioning}

Although the navigation message contents have been shown to be identical on both WAAS frequencies, which can be continuously tracked on PRN 138, 24 hour-long tests with WAAS L1 or WAAS L5 were compared against a GPS with CS to quantify and compare their impacts. These three tests were run on different days with the conditions defined in Table 5.

Satellites may fail to maintain coherency between the broadcast code and carrier. However, this threat has never been observed on the GPSL1 signal, but has been observed on WAAS geostationary satellite signals (over 24-hour cycles) and on the GPS L5 signal. Because of different receiver averaging windows, this error cannot be differentially corrected [4]. Nevertheless, L1 vs. L5 Code-Carrier Coherence (CCC) can be mitigated using Code Minus Carrier corrected for the Ionosphere (CMCI) [15]. Anyhow, only GPS L1 C/A pseudoranges are used in the solution, the CCC error associated with this diurnal variation should thus not impact the performance comparison presented in Table 6 (horizontal 2D error relative to the static antenna position obtained with postprocessed RTK) and Table 7 (improvements obtained for the different WAAS solutions compared to that of GPS CS). These augmented solutions mean errors may seem very high compared to other performance evaluations and is thus only used as a relative comparison. It can be seen that there are important improvements of both WAAS solutions over GPS CS. However, the differences between the two WAAS solutions are not noticeable because the order of magnitude difference is only a few centimeters, which could be attributed to external causes such as diurnal variations.

Hence, these observations corroborate the current WAAS implementation where both WAAS frequencies currently transmit exactly the same data. However, the message content on L5 is expected to be revised as part of the dual frequency operations capability. Meanwhile, since the overall performances are comparable for both

Table 4. Static position performances with WAAS corrections.

\begin{tabular}{cccc}
\hline & \multicolumn{2}{c}{ Probability Circles (m) } \\
\cline { 3 - 4 }$\left(\mathrm{dB} / \mathrm{N}_{0}\right.$ & $\mathrm{R}^{\mathrm{a}}$ & $\mathrm{CEP}^{\mathrm{b}}$ \\
\hline 135 & $\sim 40.0$ & 1.35 & 0.45 \\
138 & $\sim 46.5$ & 1.38 & 0.48 \\
\hline
\end{tabular}

a. R95 stands for 95\% Radius. b. CEP stands for Circular Error Probability (at 50\%).

Table 5. Characteristic of the solutions obtained with WAAS corrections from satellite PRN 138 (24H).

\begin{tabular}{|c|c|c|c|c|}
\hline Solution & Date & $\begin{array}{l}\text { Meteorological } \\
\text { observation [35] }\end{array}$ & $\begin{array}{c}\text { RxGNSS channels } \\
\text { configuration }\end{array}$ & $\begin{array}{c}\text { WAAS } \\
\mathrm{C} / \mathrm{N}_{0}(\mathrm{~dB}-\mathrm{Hz})\end{array}$ \\
\hline GPS CS & June 19, 2012 & $\begin{array}{l}\text { Overcast } 25^{\circ} \mathrm{C} \\
\text { clear visibility }\end{array}$ & -12 GPS L1 C/A & N/A \\
\hline GPS CS-WAAS L1 & June 21, 2012 & $\begin{array}{l}\text { Scattered } 28^{\circ} \mathrm{C} \\
\text { clear visibility }\end{array}$ & $\begin{array}{l}-11 \text { GPS L1 C/A } \\
-1 \text { WAAS L1 }\end{array}$ & $\begin{array}{l}\text { N/A } \\
41.0\end{array}$ \\
\hline GPS CS-WAAS L5 & June 22, 2012 & $\begin{array}{l}\text { Broken } 23.5^{\circ} \mathrm{C} \\
\text { clear visibility }\end{array}$ & $\begin{array}{l}-11 \text { GPS L1 C/A } \\
-1 \text { WAAS L5 }\end{array}$ & $\begin{array}{l}\mathrm{N} / \mathrm{A} \\
46.5\end{array}$ \\
\hline
\end{tabular}


Table 6. Solution horizontal statistics (GPS, WAAS L1 and WAAS L5 solutions).

\begin{tabular}{cccccccccc}
\hline & \multicolumn{3}{c}{ Standard deviation $(\mathrm{m})$} & \multicolumn{3}{c}{ Mean error $(\mathrm{m})$} & \multicolumn{3}{c}{ Probability circle (m) } \\
\cline { 2 - 10 } & North & East & Height & North & East & Height & CEP & R95 \\
\hline GPS CS & 1.183 & 1.007 & 2.747 & 0.681 & -0.206 & 6.470 & 0.795 & 2.434 \\
WAAS L1 & 0.809 & 0.481 & 1.568 & 0.191 & -0.105 & 2.006 & 0.475 & 1.367 \\
WAAS L5 & 0.792 & 0.488 & 1.533 & 0.269 & -0.124 & 1.740 & 0.482 & 1.376 \\
\hline
\end{tabular}

Table 7. Solution horizontal improvement statistics (WAAS solutions over GPS CS).

\begin{tabular}{cccccccccc}
\hline & \multicolumn{3}{c}{ Standard deviation (m) } & & \multicolumn{2}{c}{ Mean error (m) } & & \multicolumn{2}{c}{ Probability circle (m) } \\
\cline { 2 - 9 } & North & East & Height & North & East & Height & CEP & R95 \\
\hline WAAS L1 & 0.374 & 0.526 & 1.179 & 0.490 & -0.101 & 4.464 & 0.320 & 1.067 \\
WAAS L5 & 0.392 & 0.518 & 1.214 & 0.412 & -0.082 & 4.730 & 0.313 \\
\hline
\end{tabular}

WAAS frequencies, the following analyses are performed with correction data only obtained from WAAS L5. Similar results can be expected for $\mathrm{L} 1$, provided its $\mathrm{C} / \mathrm{N}_{0}$ is strong enough to allow continuous tracking. The next paragraph quantifies the ionospheric positioning error.

\subsection{Day vs. Night WAAS L5 Impact}

It is known that the atmosphere ions interfere more with the signals during the day, when they are polarized by the sun [36]. In order to estimate the greatest contribution of WAAS corrections further, 8-hour subsets of the previous solutions are analyzed; approaching the solar solstice,16 hours of sun and 8 without are expected. Analyzing the impact of ionospheric corrections for a single location over eight hour periods does not allow for general conclusions, as very different result could be obtained for different times and locations. Nevertheless, the intent here is to weight their relative contributions to the augmented solution considered above, i.e. a day vs. night comparison at the same location within a given 24-hour period. Hence, “day” test was extracted from June 22, 20128 A.M. to 4 P.M. (broken sky conditions at $25^{\circ} \mathrm{C}$ ) while the "night” test was extracted from 9 P.M. to 5 A.M. (scattered sky at $21.5^{\circ} \mathrm{C}$ ). Table 8 presents the improvements obtained for different WAAS solutions over GPS CS. As expected, GPS CS performs better at night than during the day, as the ionospheric delays are less harmful. Despite these results, WAAS corrections always improve the solution, but with a greater impact during the day. This allows assessing the ionospheric delays as the greatest threat, accounting for $\sim 30 \mathrm{~cm}$ of CEP. The next paragraph looks at the impact of slow and fast corrections

\subsection{Impact of Slow and Fast Corrections}

It has been shown that fast corrections needed to be applied on top of the slow corrections for them to be significant [23]. With SA discontinued, it is interesting to validate these corrections impact as they now uniquely apply to the real residual satellite clock error, i.e. the difference between the time of the actual clock and the one already modelled by the GPS Control Segment (CS) [37]. To measure the magnitude of their contribution, a 24hour test was run on July $11^{\text {th }}$, 2012 at 7h15, a day with a few clouds, clear visibility and an average temperature of $23.5^{\circ} \mathrm{C}$ [35]. Table 9 presents detailed performance metrics and improvements achieved by applying the WAAS L5 corrections to the GPS L1 C/A solution with fast corrections over the one without. Although fast corrections magnitude is not as big as for ionospheric ( 30 cm CEP) or slow corrections ( 15 cm CEP), they still provide a solution improvement ( $3 \mathrm{~cm} \mathrm{CEP})$.

\section{Discussion}

It has been shown that one $1 \mathrm{kSps}$ sample out of two is identical on both WAAS frequencies. This would also wisely provide a true message frequency diversity feature in the WAAS design, i.e. a frequency redundancy as proposed in [38], useful in case of RF interference in one of the used bands, were a WAAS L5-only capability 
Table 8. Standard deviation \& mean error improvements (Day and Night WAAS L5 over GPS CS).

\begin{tabular}{cccccccccc}
\hline & \multicolumn{3}{c}{ Standard deviation (m) } & & \multicolumn{2}{c}{ Mean error (m) } & \multicolumn{2}{c}{ Probability circle (m) } \\
\cline { 2 - 8 } & North & East & Height & North & East & Height & CEP & R95 \\
Nay & 0.464 & 0.475 & 0.952 & 0.721 & 0.081 & 5.514 & 0.453 \\
Night & 0.409 & 0.179 & 1.183 & 0.101 & -0.002 & 3.840 & 0.125 \\
\hline
\end{tabular}

Table 9. Solution horizontal statistics (with and without Fast corrections).

\begin{tabular}{cccccccccc}
\hline \multirow{2}{*}{$\begin{array}{c}\text { Fast } \\
\text { corrections }\end{array}$} & \multicolumn{3}{c}{ Standard deviation (m) } & \multicolumn{3}{c}{ Mean error (m) } & \multicolumn{3}{c}{ Probability circle (m) } \\
\cline { 2 - 9 } & North & East & Height & North & East & Height & CEP & R95 \\
\hline Yes & 0.792 & 0.488 & 1.533 & 0.269 & -0.124 & 1.740 & 0.482 & 1.376 \\
No & 0.959 & 0.524 & 1.757 & 0.161 & -0.115 & 1.561 & 0.509 & 1.663 \\
Gain & 0.168 & 0.035 & 0.224 & -0.108 & 0.009 & -0.179 & 0.027 & 0.287 \\
\hline
\end{tabular}

provided. Ultimately, the L5 broadcast will provide a new service and require different messages. Anyhow, the current required MOPS message loss rate is sufficiently low as to offer little benefit from repeating messages on both frequencies. Thus, this important advantage will be partly or totally lost when the L5 WAAS message will evolve differently from the L1 WAAS message. An alternative would be to upgrade the current WAAS L1 message to support integrity and precision on both L1 and L5, while still transmitting a duplicate of that message on WAAS L5. This would however imply to drop the SBAS service provider objective for backward compatibility with legacy L1—only users.

In terms of robustness, the WAAS signal to be tracked should be the one with the greatest $\mathrm{C} / \mathrm{N}_{0}$ available, which may not correspond to the one with the greatest elevation angle, as prescribed in the MOPS [23]. It can also be concluded that, granted such a WAAS signal is being tracked; an additional WAAS signal would not cause a positive impact on the GPS L1 C/A PVT in terms of the navigation message as all WAAS signals achieve the same performances. Nevertheless, in terms of noise performance and mitigating multipath effects, the WAAS L5 higher chipping rate sharpens the auto-correlation peak by a factor of ten, making it a better signal than WAAS L1 from a raw pseudorange standpoint. It also appears that missing a data block announcing the new indices of the upcoming data blocks does not induce noticeable differences (except maybe at cold start); either way, it could be circumvented if the indices were overlooked and RRC were applied, although this behavior is not supported by the MOPS [5].

This paper does not assess whether WAAS pseudorange should be used in the solution as this was already presented in previous work [13] [39]. This being said, despite their lower efficiency, WAAS L1 observables could be included into the solution in severe environments or when too few satellites are visible [24]; this would come at a lower cost than for WAAS L5, as the same sampling rate and front-end could be used, although WAAS L5 would indeed be a more promising alternative with its higher signal strength and lower DLL noise.

Until Direct RF Sampling as well as Software Defined Radio (SDR) become widely accessible through lower price, it may not be worthwhile to support a second front-end solely for WAAS L5, especially since only four GPS satellites are currently broadcasting on that band. The only exception to this would be for Safety of Life (SoL) applications, such as in the flight industry where high reliability is critical as lives are at stake. For such services, as well as for automated agriculture and car guidance applications, WAAS L5 offers a positive impact over $\mathrm{L} 1$ in terms of robustness due to its higher $\mathrm{C} / \mathrm{N}_{0}$, avoiding scarce discontinuities. All of this, while providing full frequency diversity in cases of signal deterioration due to jamming or unintentional interference, as long as L1 and L5 WAAS messages will remain the same.

\section{Conclusions}

In this paper, most results were related to the WAAS signal characteristics, the navigation message and the integration of the provided corrections, as opposed to previous work focused on the inclusion of WAAS pseudoranges in the navigation solution found in [13] [39], or to the WAAS L5 used in a L5-only PVT solution in [16]. 
It has been confirmed that, for the time being, WAAS navigation messages are identical on both satellite frequencies, at the time the experiments were conducted (i.e. 2012). On top of different spreading codes and rates, WAAS L5 uses Manchester coding to provide sufficient density of transitions to ensure low receiver clock drift likelihood due to lengthy strings of constant bits, while also avoiding data ambiguity; i.e. the spreading code period matches that of the transmitted symbol, thus enhancing symbol synchronization in the receiver [38]. It was also demonstrated that the broadcasted information was strongly correlated from one satellite to another and offered similar positioning performances, assuming the same tracking conditions. Speaking of which, WAAS PRN 138 provides a higher signal power on L5 over L1 by 5.5 dB-Hz, which is enough to avoid the isolated tracking losses witnessed with other WAAS satellites. Nevertheless, measured $\mathrm{C} / \mathrm{N}_{0}$ from the three WAAS satellites was not proportional to their elevation, as seen in Table 2, differing from previous findings [24]. Another displayed metric is that the WAAS L1 signal quality, in terms of pseudorange noise, is as good as for GPS L1 C/A, for the same C/ $\mathrm{N}_{0}$. WAAS L5 PRN 138 pseudorange noise is 10 times lower than that of GPS L1 C/A (at the same $\mathrm{C} / \mathrm{N}_{0}$ ), in conformity with GPS signals [32] [33].

For single frequency receivers, WAAS L1 provides a 50\% error reduction, distributed over the ionospheric, slow and fast corrections in a decreasing order of magnitude. A greater impact of WAAS corrections was noticed during the day, although WAAS corrections are still beneficial during the night, due to slow and fast corrections being required all around the clock. Hence, even if mobile devices may need to rationalize their power consumption, a single WAAS channel should be kept active at all times, although a reduced set of features could be considered.

The results presented herein should remain valid until the year 2020. By then, all GPS satellites should broadcast the GPS L5 and new L1C MBOC civil signals, while the GALILEO constellation, providing E1 MBOC and E5A signals among others, should be fully operational. WAAS L5 should then be updated to better support these new signals, hopefully preserving frequency diversity.

Up-coming research will continue assessing WAAS signals' improvement as they are deployed during Phase 4.

\section{References}

[1] Braff, R. and Shively, C. (1986) GPS Integrity Channel. Navigation, Journal of the Institute of Navigation, 32, 334-350. https://ion.org/publications/abstract.cfm?articleID=100431

[2] Kim, E., Walter, T. and Powell, J.D. (2006) Optimizing WAAS Accuracy/Stability for a Single Frequency Receiver. Proceedings of the 19th International Technical Meeting of the Satellite Division of the Institute of Navigation (ION GNSS 2006), Fort Worth, September 2006, 962-970.

[3] National Coordination Office for Space-Based Positioning Navigation and Timing (2012) Selective Availability. http://www.gps.gov/systems/gps/modernization/sa/

[4] Walter, T., Blanch, J., Phelts, R.E. and Enge, P. (2012) Evolving WAAS to Serve L1/L5 Users. Navigation, 59, 317327. http://dx.doi.org/10.1002/navi.21

[5] RTCA (2006) Minimum Operational Performance Standards for Global Positioning System/Wide Area Augmentation System Airborne Equipment. Document Number: DO-229D.

[6] Lawrence, D., Bunce, D., Mathur, N.G. and Sigler, C.E. (2007) Wide Area Augmentation System (WAAS)—Program Status. Proceedings of the 20th International Technical Meeting of the Satellite Division of the Institute of Navigation (ION GNSS 2007), The Institute of Navigation, 892-899.

[7] Hathaway, D.H. (2012) The Sunspot Cycle. http://solarscience.msfc.nasa.gov/SunspotCycle.shtml

[8] Gannon, M. (2013) Sun’s 2013 Solar Activity Peak Is Weakest in 100 Years. Online. http://www.space.com/21937-sun-solar-weather-peak-is-weak.html

[9] Federal Aviation Administration (2012). Navigation Services-WAAS—News. http://www.faa.gov/about/office_org/headquarters_offices/ato/service_units/techops/navservices/gnss/waas/news/

[10] Fernandez-Prades, C., Presti, L.L. and Falletti, E. (2011) Satellite Radio Localization from GPS to GNSS and Beyond: Novel Technologies and Applications for Civil Mass Market. Proceedings of the IEEE, 99, 1882-1904. http://dx.doi.org/10.1109/JPROC.2011.2158032

[11] Jan, S.-S. (2002) Analysis of a Three-Frequency GPS/WAAS Receiver to Land an Airplane. Proceedings of the 15th International Technical Meeting of the Satellite Division of the Institute of Navigation ION GPS 2002, Oregon Convention Center, Portland, 24-27 September, 2002, 2576-2586.

[12] Murfin, T. (2012) ION Nashville 2012 Round-Up. GPS World. 
http://www.gpsworld.com/ion-nashville-2012-round-up/

[13] Rho, H. and Langley, R.B. (2008) Evaluation of the New WAAS L5 Signal. Proceedings of ION GNSS 21st International Technical Meeting of the Satellite Division of the Institute of Navigation, Savannah, 16-19 September 2008, 1667-1678.

[14] Hsu, P.-H., Cheung, L. and Grewal, M. (2004) Prototype Test Results of L1/L5 Signals of Future GEO Satellites. Proceedings of ION GNSS 17th International Technical Meeting of the Satellite Division, Long Beach, 21-24 September, 1359-1366.

[15] Federal Aviation Administration (2011) WAAS Technical Specifications for GEO 5/6/7 Service Lease. Department of Transportation, Vol. WAAS100232, 45.

[16] Chen, Y.H., Juang, J.C., De Lorenzo, D.S., Seo, J., Lo, S., Enge, P., et al. (2011) Real-Time Dual-Frequency (L1/L5) GPS/WAAS Software Receiver. Proceedings of the 24th International Technical Meeting of the Satellite Division of the Institute of Navigation (ION GNSS 2011), Oregon Convention Center, Portland, Oregon, 20-23 September 2011, 767-774.

[17] Federal Aviation Administration (2008) Global Positionning System Wide Area Augmentation System (WAAS) Performance Standard. 1st Edition, Department of Transportation, 28.

http://www.gps.gov/technical/ps/2008-WAAS-performance-standard.pdf

[18] Landry, R.J., Fortin, M.A. and Guay, J.C. (2010) Universal Acquisition and Tracking Apparatus for Global Navigation Satellite System (GNSS). Canada Patent No 12/767,773.

[19] Fortin, M.A., Guay, J.C. and Landry, R.J. (2009) Development of a Universal GNSS Tracking Channel. Proceedings of the 22nd International Technical Meeting of the Satellite Division of the Institute of Navigation ION GNSS 2009, Savannah, GA, 22-25 September 2009, 259-272.

[20] Fortin, M.A., Guay, J.C. and Landry, R.J. (2009) Real-Time Low-Cost Multipath Mitigation Technique Calibrated through Real Data Repeatable Testing. Proceedings of the 22nd International Technical Meeting of the Satellite Division of the Institute of Navigation ION GNSS 2009, Savannah, Georgia, 22-25 September 2009, 2316-2328.

[21] Guay, J.C., Sauriol, B. and Fortin, M.A. (2008) Real-Time Multipath Monitoring and Characterization with a Variable Spacing Correlator on a FPGA-Based Software GNSS Receiver. Proceedings of the 21st International Technical Meeting of the Satellite Division of the Institute of Navigation ION GNSS 2008, Savannah, 16-19 September 2008, 23322342.

[22] Sauriol, B. and Landry, R.J. (2007) FPGA-Based Architecture for High Throughput, Flexible and Compact Real-Time GNSS Software Defined Receiver. Proceedings of the 2007 National Technical Meeting of the Institute of Navigation, San Diego, 22-24 January 2007, 708-717.

[23] Radio Technical Commission for Aeronautics Special Committee 159 (2001) Minimum Operational Performance Standards for Global Positioning System/Wide Area Augmentation System Airborne Equipment. RTCA. http://www.rtca.org/store product.asp?prodid=817

[24] Langley, R.B. and Rho, H. (2009) Innovation: The WAAS L5 Signal. GPS World. http://www.gpsworld.com/gnss-system/innovation-the-waas-l5-signal-7047

[25] Issler, J.L., Ries, L., Bourgeade, J.M., Lestarquit, L. and Macabiau, C. (2004) Probabilistic Approach of Frequency Diversity as Interference Mitigation Means. Proceedings of the 17th International Technical Meeting of the Satellite Division of the Institute of Navigation (ION GNSS 2004), Long Beach, CA, 21-24 September 2004, 2136-2145.

[26] Guay, J.C. (2010) Récepteur SBAS-GNSS logiciel pour des applications temps-réel. École de technologie supérieure, Montréal.

[27] Todd, K.M. (2005) Error Correction Coding: Mathematical Methods and Algorithms. Wiley Series Edition, John Wiley \& Sons Inc., New Jersey. ISBN: 978-0-471-64800-0.

[28] Federal Aviation Administration (2001) Specification for the Wide Area Augmentation System (WAAS). Department of Transportation, 156.

http://www.faa.gov/about/office_org/headquarters_offices/ato/service_units/techops/navservices/gnss/library/document s/media/waas/2892bC2a.pdf

[29] Julien, O., Macabiau, C., Issler, J.L., Nouvel, O. and Vigneau, W. (2006) Analysis and Quality Study of GNSS Monitoring Ground Stations' Pseudorange and Carrier-Phase Measurements. Proceedings of the 19th International Technical Meeting of the Satellite Division of The Institute of Navigation (ION GNSS 2006), Fort Worth Convention Center, Fort Worth, TX, 26-29 September 2006, 971-980.

[30] Betz, J.W. (2000) Design and Performance of Code Tracking for the GPS M Code Signal. Proceedings of the 13th International Technical Meeting of the Satellite Division of the Institute of Navigation ION GNSS 2000, Salt Lake City, UT, 19-22 September 2000, 2140-2150.

[31] Ries, L., Lestarquit, L., Armengou-Miret, E., Legrand, F., Vigneau, W., Bourga, C., Erhard, P. and Issler, J.L. (2002) A 
Software Simulation Tool for GNSS2 BOC Signals Analysis. Proceedings of the 15th International Technical Meeting of the Satellite Division of the Institute of Navigation ION GNSS 2002, Institute of Navigation, Portland, OR, 24-27 September 2002, 2225-2239.

[32] ARINC Engineering Services (2005) Navstar GPS Space Segment/User Segment L5 Interfaces. Interface Specification, Navstar GPS Joint Program Office, El Segundo, California, 95.

[33] Global Positioning System Wing (GPSW) Systems Engineering \& Integration (2010) Navstar GPS Space Segment/ Navigation User Interfaces. Los Angeles Air Force Base, El Segundo, California, 185. http://www.losangeles.af.mil/shared/media/document/AFD-100813-045.pdf

[34] Federal Aviation Administration (2012) Satellite Position and WAAS Status. http://www.nstb.tc.faa.gov/RT_WaasSatelliteStatus.htm

[35] Oolman, L. (2012) Surface Observations. http://weather.uwyo.edu/surface/meteorogram/

[36] El-Rabbany, A. (2002) Introduction to GPS: The Global Positoning System. Mobile communications Series, Boston.

[37] Jan, S.S. (2003) Aircraft Landing Using a Modernized Global Positioning System and the Wide Area Augmentation System. Doctor of Philosophy Thesis, Aeronautics and Astronautics, Stanford University, Stanford.

[38] Van Dierendonck, A.J., Hegarty, C., Niles, R., Morrissey, T. and Reddan, P. (2005) Next Generation Satellite Based Augmentation System (SBAS) Signal Specification. Proceedings of the 2005 National Technical Meeting of the Institute of Navigation, San Diego, CA, 24-26 January 2005, 371-384.

[39] Schempp, T., Burke, J. and Rubin, A. (2008) WAAS Benefits of GEO Ranging. 21st International Technical Meeting of the Satellite Division of the Institute of Navigation ION GNSS 2008, Savannah, GA, 16-19 September 2008, 20002007. 
Scientific Research Publishing (SCIRP) is one of the largest Open Access journal publishers. It is currently publishing more than 200 open access, online, peer-reviewed journals covering a wide range of academic disciplines. SCIRP serves the worldwide academic communities and contributes to the progress and application of science with its publication.

Other selected journals from SCIRP are listed as below. Submit your manuscript to us via either submit@scirp.org or Online Submission Portal.
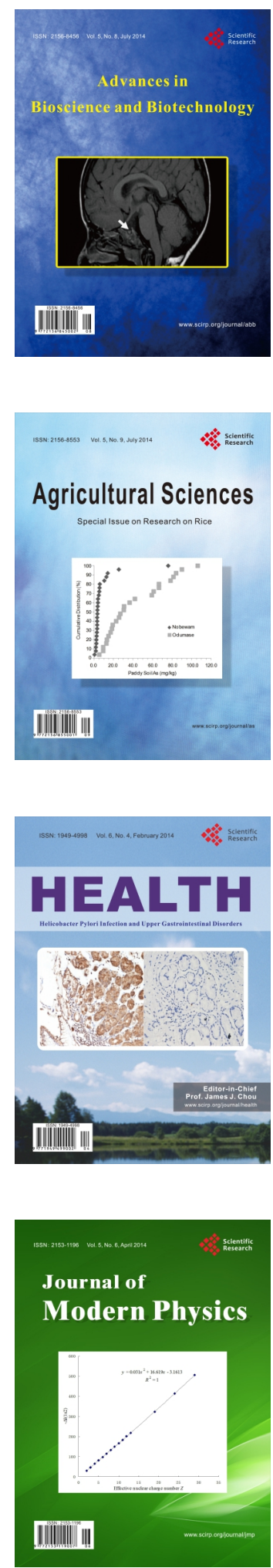
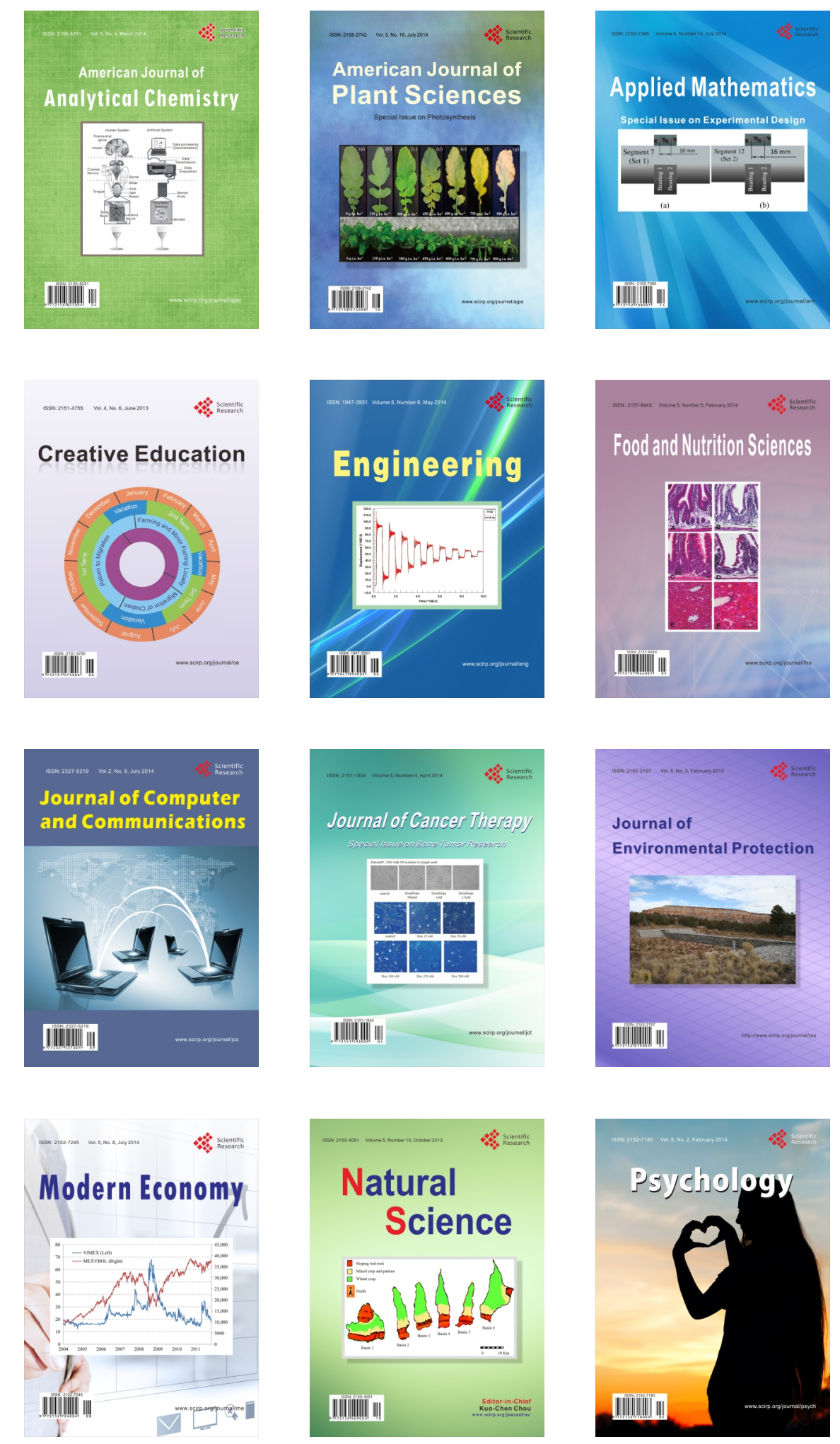